\title{
Tenascin-C expression and its associated pathway in BMSCs following co-culture with mechanically stretched ligament fibroblasts
}

\author{
BING ZHAO $^{1}$, MENGCAI HU ${ }^{1}$, HUIYAN WU ${ }^{1}$, CHENCHEN REN $^{1}$, JIANSHE WANG ${ }^{2}$ and SHIHONG CUI ${ }^{1}$ \\ ${ }^{1}$ Department of Obstetrics, The Third Affiliated Hospital of Zhengzhou University, Zhengzhou, Henan 450052; \\ ${ }^{2}$ Department of Clinical Medicine, Hebi Polytechnic College, Hebi, Henan 458030, P.R. China
}

Received January 13, 2016; Accepted January 9, 2017

DOI: $10.3892 / \mathrm{mmr} .2017 .6329$

\begin{abstract}
The occurrence of pelvic organ prolapse (POP) is closely associated with alterations in the extracellular matrix proteins of the supporting ligament. Bone marrow mesenchymal stem cells (BMSCs) have the potential to differentiate into a variety of cell types, including osteoblasts, chondroblasts and adipocytes. Therefore, BMSCs have the potential to improve the clinical outcomes of POP. Tenascin- $\mathrm{C}$ is a large glycoprotein that is present in the ECM and is involved in morphogenetic movements, and tissue patterning and repair. The aim of the present study was to investigate the effect of mechanical stretching on tenascin- $\mathrm{C}$ expression during the differentiation of BMSCs induced by pelvic ligament fibroblasts. BMSCs were isolated from 7-day-old Sprague Dawley rats. Fibroblasts were obtained from rat pelvic ligaments and, at the fourth passage, were subjected to $10 \%$ deformation with $1 \mathrm{~Hz}$, periodic one-way mechanical stretch stimulation, followed by co-culture with BMSCs. The co-culture with stretched fibroblasts increased tenascin- $\mathrm{C}$ and transforming growth factor (TGF)- $\beta$ expression levels, compared with groups without mechanical stimulation. Neutralizing anti-TGF- $\beta 1$ antibodies, and inhibitors of TGF- $\beta$ receptor, mitogen-activated protein kinase (MAPK) kinase and MAPK, decreased tenascin- $C$ expression levels induced by TGF- $\beta$ and mechanical stretching. The results of the present study suggested that the regulation of tenascin- $C$ expression levels in BMSCs co-cultured with mechanically stretched pelvic ligament fibroblasts is mediated via the soluble growth factor TGF- $\beta$ and the MAPK signaling pathway. In addition, these results indicated that in an indirect co-culture system, pelvic ligament fibroblasts with mechanical stretch stimulation may
\end{abstract}

Correspondence to: Dr Shihong Cui, Department of Obstetrics, The Third Affiliated Hospital of Zhengzhou University, 7 Kangfu Front Street, Erqi, Zhengzhou, Henan 450052, P.R. China

E-mail: cuishihongchina@gmail.com

Key words: bone marrow stromal cells, stretch, differentiation, tenascin-C, pelvic ligament fibroblasts promote the synthesis of tenascin- $\mathrm{C}$ and BMSC differentiation into pelvic ligament fibroblasts.

\section{Introduction}

The incidence of pelvic organ prolapse (POP) is increasing in aging populations due to the prolonged life span following the menopause $(1,2)$, and is becoming one of the most common forms of female pelvic floor dysfunction. POP causes various complications and significantly affects the quality of life of patients (3). The occurrence of POP is closely associated with elasticity, toughness and functional alterations in the connective tissue of the pelvic support tissue (4). Normal pelvic structure support and function depend on the pelvic floor connective tissues. It has previously been reported that alterations in extracellular matrix (ECM) proteins of the supporting ligament are associated with POP (5).

Bone marrow mesenchymal stem cells (BMSCs) originate from the mesoderm, have multilineage capacity and generate a variety of cell types (6). BMSCs may induce and regulate the development of bone marrow hematopoietic stem cells and stromal cells. In addition, they may promote the assembly and ordered arrangement of ECM molecules (7). Following injury, endogenous or exogenous BMSCs migrate into lesions for repair. Increasing evidence suggests that in the process of ligament injury repair, different pluripotent stem cells are released from bone marrow. These stem cells are similar to the surrounding ligament cells, and move to and accumulate in the injury sites $(8,9)$. Young et al studied BMSCs in vivo (10). Following replacement of the Achilles tendon of rabbits with collagen fibers and BMSCs, assembly of the fibers was improved compared with replacement with collagen fibers only. The freshly produced ligament fibers were thicker compared with the original (9). In a separate study, BMSCs or fibroblasts in a liquid fibrin matrix were injected into the damaged knee tendon and patellar tendon of immunodeficient rats. This study reveals that an injection of BMSCs or fibroblasts in a liquid fibrin matrix altered tissue morphology, ultrastructure and the mRNA expression levels of ECM proteins, which facilitate damage repair (11). Therefore, BMSCs have the potential to improve recovery from injury. 
Tissue repair depends on intrinsic and extrinsic processes, requiring blood supply, fibroblasts, ECM proteins and growth factors for proliferation, repair and remodeling (12). In connective tissue, evidence suggests that constant mechanical stress regulates the synthesis of ECM proteins, including collagen, elastin, tenascin and integrin ligands, and their arrangement (13). These ECM proteins regulate mechanical signal transduction and promote the secretion of growth factors (14). Simultaneously, via integrins, mechanical stress signals are transmitted to the cytoskeleton to assemble in a certain physiological range and pattern of dynamics (15). Transforming growth factor (TGF)- $\beta$ is a known stimulator of ECM protein production in fibroblasts and mediates the response of fibroblasts to mechanical stress (16). Tenascin-C is an ECM component that is upregulated in fibroblasts following mechanical stress stimulation. In addition, tenascin- $\mathrm{C}$ is highly expressed during wound healing and tissue remodeling (17-19). However, little is known regarding the tenascin- $\mathrm{C}$ pathway during BMSC differentiation.

Therefore, the present study aimed to determine the effect of pelvic ligament fibroblasts following stretch stimulation on tenascin- $\mathrm{C}$ expression and its pathway in BMSCs, using a co-culture system. This may facilitate further understanding of BMSC differentiation potential and characteristics, which may aid in the development of novel therapeutic strategies for the treatment of POP.

\section{Materials and methods}

Experimental animals and primary reagents. Female, 7-week-old Sprague Dawley rats ( $=20 ; 225-275 \mathrm{~g})$ were purchased from the Experimental Animal Center of The Fourth Military Medical University (Xi'an, China). They were maintained under controlled conditions of $22-26^{\circ} \mathrm{C}, 12 \mathrm{~h}$ light/dark cycle and a relative humidity of $50-70 \%$. Animals had access to food and water throughout the experiment. All studies were conducted in accordance with the standards of humane animal care described in the National Institutes of Health Guide for the Care and Use of Laboratory Animals (20), using protocols approved by Zhengzhou University Institutional Animal Care and Research Advisory Committee (Zhengzhou, China). Low glucose Dulbecco's modified Eagle's medium (LG-DMEM) and trypsin were purchased from Gibco; Thermo Fisher Scientific, Inc. (Waltham, MA, USA), fetal bovine serum (FBS) was obtained from Hangzhou Sijiqing Biological Engineering Materials Co., Ltd. (Hangzhou, China). Penicillin and streptomycin sulfate were purchased from North China Pharmaceutical Group Co., Ltd. (Shijiazhuang, China), and Percoll separation medium was purchased from Pfizer, Inc. (New York, NY, USA). For flow cytometry, the mouse anti-rat monoclonal antibodies cluster of differentiation (CD)44-fluorescein isothiocyanate (FITC; cat. no. 561859), CD90-phycoerythrin (PE; cat. no. 551401), CD45-FITC (cat. no. 551401), and the isotype control antibodies mouse anti-rat IgG1-FITC (cat. no. 553892) and mouse IgG1-PE (cat. no. 554680) were obtained from BD Biosciences (Franklin Lakes, NJ, USA); the mouse anti-rat CD34-FITC monoclonal antibody (cat. no. sc-7324 FITC) was purchased from Santa Cruz Biotechnology, Inc., (Dallas, TX, USA). The Total Protein assay kit was purchased from Beyotime Institute of
Biotechnology (Shanghai, China). The following antibodies were used for western blotting and immunofluorescence: Alexa Fluor ${ }^{\circledR} 555$ Phalloidin (cat. no. 8953; Cell Signaling Technology, Inc., Danvers, MA, USA); mouse anti-rat F-actin antibody (cat. no. ab205, Abcam, Cambridge, UK); mouse anti-rat GAPDH antibody (cat. no. SC-47724; Santa Cruz Biotechnology, Inc.); rabbit anti-rat tenascin-C antibody (cat. no. SC-20932; Santa Cruz Biotechnology, Inc.); goat anti-rabbit IgG-FITC (cat. no. SC-2012; Santa Cruz Biotechnology, Inc.); horseradish peroxidase-conjugated goat anti-mouse IgG secondary antibodies (cat. no. 1706516; Bio-Rad Laboratories, Inc., Hercules, CA, USA) and anti-rabbit IgG secondary antibodies (cat. no. 1706515; Bio-Rad Laboratories, Inc.). One-Step SYBR PrimeScript RT-PCR kit II was obtained from Takara Biotechnology Co., Ltd., (Dalian, China) for reverse transcription-quantitative polymerase chain reaction (RT-qPCR).

Isolation and analysis of rat BMSCs. Isolation, culture and passage of rat BMSCs from 7-day-old Sprague Dawley rats were performed as previously described (21) following sacrifice of rats by cervical dislocation. Briefly, under sterile conditions, a syringe was used to flush out the bone marrow. Bone marrow cells were isolated with Percoll separation medium. The interface layer of mononuclear cells was washed twice with cold PBS and maintained in complete LG-DMEM (10\% FBS; $100 \mathrm{U} / \mathrm{ml}$ penicillin and $100 \mathrm{mg} / \mathrm{ml}$ streptomycin sulfate). These primary cells were subcultured at $80-90 \%$ confluence. Fourth passage BMSCs were used for experimental analysis.

Flow cytometry was performed to determine the surface expression of CD44, CD90, CD45 and CD34 on rat BMSCs. On reaching $80-90 \%$ confluence, fourth passage BMSCs $\left(1 \times 10^{5}\right.$ cells $\left./ \mathrm{ml}\right)$ were stained with $1 \mu \mathrm{l} \mathrm{CD} 44-F I T C ~(1: 1,000)$, CD90-PE (1:1,000), CD45-FITC (1:1,000), CD34-FITC $(1: 1,000)$, IgGl-FITC $(1: 1,000)$ or IgGl-PE $(1: 1,000)$ at room temperature in the dark for $30 \mathrm{~min}$. The labeled cells were centrifuged at $208 \times \mathrm{g}$ for $5 \mathrm{~min}$ at $4^{\circ} \mathrm{C}$ and resuspended in PBS for analysis using a FACScan flow cytometer (BD Biosciences). CellQuest $^{\mathrm{TM}}$ Pro Version 4.0 (BD Biosciences) was used for analysis. As described previously (4), the control cells were cultured in complete medium (LG-DMEM; 10\% FBS; $100 \mathrm{U} / \mathrm{ml}$ penicillin and $100 \mathrm{mg} / \mathrm{ml}$ streptomycin sulfate) and the treatment group was cultured with osteogenic and adipogenic induction, respectively. After 12 and 14 days, cells were fixed with paraformaldehyde and stained with alizarin red or Oil-red-O solution for observation under microscope $(22,23)$.

Growth factors and inhibitors. SB 431542 (a TGF- $\beta$ receptor type I inhibitor), PD 98059 [a mitogen-activated protein kinase (MAPK) kinase (MEK-1) inhibitor] and SB 203580 (a p38 MAPK inhibitor) were purchased from EMD Millipore (Billerica, MA, USA). Stock solutions of SB 431542, PD 98059 and SB 203580 were prepared in dimethyl sulfoxide (DMSO). Neutralizing anti-TGF- $\beta 1$ antibodies were obtained from R\&D Systems, Inc. (Minneapolis, MN, USA). Human recombinant TGF- $\beta 1$ was purchased from Sigma-Aldrich (Merck KGaA, Darmstadt, Germany) and prepared in bovine serum albumin (BSA; Sigma-Aldrich; Merck KGaA)-containing stock solutions according to the manufacturer's protocol. Growth factor and inhibitor concentrations used in assays were optimized 
based on the literature (24-26), our preliminary experiments and the manufacturer's data sheets, and the following final concentrations were selected: Neutralizing anti-TGF- $\beta 1$ antibodies, $100 \mathrm{ng} / \mathrm{ml}$; SB 431542, $10 \mu \mathrm{M}$; PD 98059, $10 \mu \mathrm{M}$; SB 203580, $3 \mu \mathrm{M}$; and TGF- $\beta 1,5 \mathrm{ng} / \mathrm{ml}$.

Rat pelvic ligament fibroblasts and fibroblast traction injury model. Rat pelvic ligament fibroblasts were prepared as previously described (21) and maintained in LG-DMEM containing $10 \% \mathrm{FBS}$, and $100 \mathrm{U} / \mathrm{ml}$ penicillin and $100 \mathrm{mg} / \mathrm{ml}$ streptomycin sulfate. Briefly, ligament was removed and immediately placed into serum-free Hanks' balanced salt solution (HBSS) and minced into $1 \mathrm{~mm}^{3}$, followed by treatment with $0.25 \%$ trypsin and $0.01 \%$ EDTA at $37^{\circ} \mathrm{C}$ for 2 days with gentle rotation. The supernatant, which contained the fibroblasts, was washed with HBSS and DMEM with $10 \%$ heat-inactivated FBS. The cell suspension was spun down twice to remove collagenase. The pellets were resuspended in growth medium and plated in tissue culture flasks. The medium was changed every other day. Prior to stretching, a small number of fourth passage fibroblasts were collected for immunofluorescence staining to confirm that they were fibroblasts. Passage 3 fibroblasts ( $3 \times 10^{5} /$ membrane) were seeded onto gelatin-coated silicone membranes and cultured for $24 \mathrm{~h}$, following culture, cells were considered to be passage 4 . Subsequently, $10 \%$ of the load transformation was exerted on cells with $1 \mathrm{~Hz}$ horizontal stretch stimulation for various durations $(0,3$, 12 or $24 \mathrm{~h}$ ) under $5 \% \mathrm{CO}_{2}$ and $37^{\circ} \mathrm{C}$ conditions. Cells were fixed in paraformaldehyde and stained with $5 \mu \mathrm{g} / \mathrm{ml}$ DAPI. Cell morphology was examined under a confocal microscope (Olympus Corporation, Tokyo, Japan). Prior to co-culture, ligament fibroblasts were subjected to mechanical stretch stimulation for $12 \mathrm{~h}$ and subsequently seeded into the upper chamber at $5 \times 10^{4} /$ well. BMSCs $\left(2 \times 10^{5} /\right.$ well $)$ were seeded into the lower chamber of a 6 -well Transwell plate. Following indirect co-culture for 3, 6 or 12 days, cells were collected for RT-qPCR and western blot analysis.

Treatment with growth factor and inhibitors. BMSCs ( $2 \times 10^{5}$ cells/well in $\left.2 \mathrm{ml}\right)$ in the lower chamber and fibroblast cells $\left(5 \times 10^{4}\right.$ cells/well in $\left.2 \mathrm{ml}\right)$ in the upper chamber were cultured in 6-well Transwell plates. Protein kinase inhibitors and growth factors were diluted with medium at double the final concentration stated previously: Neutralizing anti-TGF- $\beta 1$ antibodies, $200 \mathrm{ng} / \mathrm{ml}$; SB 431542, $20 \mu \mathrm{M}$; PD 98059, $20 \mu \mathrm{M}$; SB 203580, $6 \mu \mathrm{M}$; and TGF- $\beta 1,10 \mathrm{ng} / \mathrm{ml}$. The control group was treated with medium containing $0.1 \%$ DMSO. Controlor inhibitor-containing medium $(2 \mathrm{ml})$ was placed into each well and incubated for $30 \mathrm{~min}$. Subsequently, $2 \mathrm{ml}$ medium with or without TGF- $\beta 1$ was added to the upper and lower compartments of each well. Cells were collected at different time points following co-culture (3, 6 or 12 days), which was followed by lysis and total RNA extraction as described below.

TGF- $\beta 1$ ELISA. TGF- $\beta 1$ was detected using a commercially available ELISA kit (cat. no. MB100B, R\&D Systems). Representative samples were analyzed for the presence of active TGF- $\beta 1$; however, the concentration was below the detection limit of the assay. Therefore, all samples were activated by acidification to separate TGF- $\beta 1$ from its binding proteins, allowing for measurement of total TGF- $\beta 1$. For activation of samples containing FBS, $2.5 \mathrm{M}$ acetic acid+10 $\mathrm{M}$ urea was used, and $1 \mathrm{M} \mathrm{HCl}$ was used for microdialysis samples (as recommended by the supplier). Activated samples were neutralized using $2.7 \mathrm{M} \mathrm{NaOH}+1 \mathrm{M}$ 4-(2-hydroxyethyl)-1-piperazinee-thanesulfonic acid (HEPES) for samples with FBS and 1.2 M NaOH+0.5 M HEPES for microdialysis (as recommended by the supplier). Samples were loaded onto ELISA plates immediately following neutralization. All samples were measured in duplicate.

$R T$ - $q P C R$. RNA was isolated using TRIzol ${ }^{\circledR}$ reagent (Takara Biotechnology Co., Ltd.). The RNA yield was determined by measuring the absorbance at a wavelength of $260 \mathrm{~nm}$. RNA was subsequently reverse-transcribed to cDNA, and the cDNA was amplified by qPCR using the $S \mathrm{YBR}^{\circledR}$-Green reporter that was included in the kit used for qPCR and the following thermocycling conditions: Pre-denaturation for $2 \mathrm{~min}$ at $94^{\circ} \mathrm{C}$; followed by 45 cycles of $30 \mathrm{sec}$ at $60^{\circ} \mathrm{C}$ and $60 \mathrm{sec}$ at $55^{\circ} \mathrm{C}$. The specificity of the produced amplification product was confirmed by examination of dissociation reaction plots. Each sample was tested in triplicate, and samples obtained from 3 independent experiments were used for analysis of relative gene expression, normalization to GAPDH expression was performed using the $\Delta \Delta \mathrm{Cq}$ method (27-29). The following primers were designed by Takara Biotechnology Co., Ltd.: GAPDH, 5'-GACATC AAGAAGGTGGTGAAGC-3' (forward) and 5'-TGTCATTGA GAGCAATGCCAGC-3' (reverse); and tenascin-C, 5'-ACC ATGCTGAGATAGATGTTCCAAA-3' (forward) and 5'-CTT GACAGCAGAAACACCAATCC-3' (reverse).

Western blot analysis. Cells were lysed using $200 \mu \mathrm{l}$ radioimmunoprecipitation assay lysis buffer (cat. no. R0278, Sigma-Aldrich; Merck KGaA). Equal quantities of proteins $(20 \mu \mathrm{g})$ were loaded onto $10 \%$ Tris-Glycine gels following denaturation. The proteins were subsequently transferred to polyvinylidene difluoride membranes. Membranes were blocked with 5\% milk in PBS containing $0.1 \%$ Tween-20 (PBST), followed by incubation with primary antibodies against F-actin $(1: 1,000)$, and GAPDH $(1: 1,000)$ at $4^{\circ} \mathrm{C}$ overnight. The membranes were washed with PBST and incubated for $1 \mathrm{~h}$ at room temperature with horseradish peroxidase-conjugated secondary antibodies $(1: 2,000)$. Following washing, protein bands were visualized using a Chemiluminescence Protein Detection kit (Pierce; Thermo Fisher Scientific, Inc.). Each experiment was repeated in triplicate. GAPDH served as the internal control.

Immunofluorescent staining. Cells (fibroblasts and BMSCs) were cultured on coverslips in a 6-well plate. Following mechanical stress or co-culture, cells were fixed with $3 \%$ paraformaldehyde at $4^{\circ} \mathrm{C}$ in PBS containing $1 \mathrm{mM}$ calcium chloride for $10 \mathrm{~min}$ and permeabilized by the use of $0.2 \%$ Triton X-100 at room temperature for $5 \mathrm{~min}$. Subsequently, cells were incubated with blocking solution (containing 5\% goat serum, $0.3 \%$ Triton X-100, and $0.1 \%$ BSA in PBS) at room temperature for $30 \mathrm{~min}$. Following 3 rinses, slides were incubated with Alexa Fluor 555 Phalloidin (1:20) for $1 \mathrm{~h}$ at $37^{\circ} \mathrm{C}$. Rinsed slides were incubated with anti-tenascin-C (1:500) primary antibody at $4^{\circ} \mathrm{C}$ overnight. After washing and the addition of goat anti-rabbit IgG-FITC (1:500) for $1 \mathrm{~h}$ at $37^{\circ} \mathrm{C}$, nuclear 
staining was performed with DAPI (1:20,000; Sigma-Aldrich; Merck $\mathrm{KGaA}$ ) for $5 \mathrm{~min}$. Slices were subsequently mounted and observed under a fluorescent microscope.

Statistical analysis. Data are expressed as the mean \pm standard deviation. Statistical significance was analyzed using one-way analysis of variance followed by Tukey's post hoc test, and was performed using SPSS software version 11.0 (SPSS, Inc., Chicago, IL, USA). $\mathrm{P}<0.05$ was considered to indicate a statistically significant difference.

\section{Results}

Isolation and characterization of BMSCs. As described in our previous studies $(21,30)$, scattered small BMSCs colonies formed, fused and stacked following several passages. Following 3-4 passages, cells exhibited more homogeneous morphology. These homogeneous cells were collected for expression profile analysis by flow cytometry. Cells expressed CD44 and CD90 (Fig. 1) and may be induced to differentiate into osteoblasts and adipogenic cells (data not shown).

Verification of the rat fibroblast stretch injury model. Fibroblasts were subjected to $10 \%$ load and $1 \mathrm{~Hz}$ stretch optimized according to our previous studies $(21,30)$ and preliminary experiments. Compared with the control group, this mechanical stretch elongated the cell body and increased shape index values at different time points (data not shown). Under a laser confocal scanning microscope, non-stretching rat ligament fibroblasts exhibited the typical morphology of polygonal cells with a thick F-actin fiber bundle (Fig. 2A). However, $24 \mathrm{~h}$ of mechanical stress decreased the F-actin staining intensity (Fig. 2B); the effect on F-actin protein expression levels was time-dependent, as demonstrated by western blotting (Fig. 2C).

Time-dependent induction of tenascin- $C$ in BMSCs by mechanically stretched fibroblasts. Preliminary experiments (data not shown) revealed that mechanical stress significantly and directly increased tenascin-C expression in fibroblasts in a time-dependent manner. However, whether indirect co-culture with mechanically stretched ligament fibroblasts may regulate tenascin-C expression in BMSCs remains controversial. Therefore, the present study used an indirect co-culture system to investigate tenascin-C expression levels and pathway in BMSCs cultured with stretched fibroblasts. Following co-culture with stretched fibroblasts, tenascin-C mRNA expression levels in BMSCs increased $\sim 1.5$-fold $(\mathrm{P}<0.05)$ after 3 days, 2.4 -fold $(\mathrm{P}<0.05)$ after 6 days and 4 - to 5-fold $(\mathrm{P}<0.05)$ after 12 days, compared with the control group (Fig. 3A). The data indicated that co-culture with mechanically stretched fibroblasts increased the tenascin-C mRNA expression levels in BMSCs in a time-dependent manner. In accordance with this, immunofluorescence staining revealed that tenascin-C expression was increased in BMSCs following co-culture with mechanically stimulated fibroblasts for 12 days, compared with control BMSCs (Fig. 3B).

Synergistic effects of TGF- $\beta$ and indirect co-culture with mechanically stretched fibroblasts on tenascin- $C$ expression levels. It was subsequently investigated whether TGF- $\beta$ treatment and co-culture with strained fibroblasts had synergistic effects. TGF- $\beta$ is a paracrine growth factor mediating the cellular response to mechanical strain. This factor induces tenascin-C mRNA expression in fibroblasts $(31,32)$. Therefore, it was hypothesized that mechanical stretching of fibroblasts may indirectly promote tenascin- $\mathrm{C}$ expression in BMSCs via the release of TGF- $\beta 1$. To investigate this possibility, BMSCs were treated with exogenous TGF- $\beta 1$ to determine whether this synergistically increased the effects of mechanical stretch on tenascin-C mRNA expression levels. With or without addition of TGF- $\beta 1$, BMSCs were cultured with mechanically stretched fibroblasts for 6 days. As presented in Fig. 4 , in the absence of TGF- $\beta 1$, mRNA expression levels of tenascin-C in BMSCs co-cultured with mechanically stimulated fibroblasts were 2-fold greater $(\mathrm{P}<0.05)$ compared with control BMSCs co-cultured with fibroblasts without mechanical stimulation. The addition of $5 \mathrm{ng} / \mathrm{ml}$ TGF- $\beta 1$ significantly increased tenascin- $\mathrm{C}$ mRNA expression levels $\sim 3.8$-fold $(\mathrm{P}<0.05)$ compared with BMSCs cultured in the absence of TGF- $\beta 1$. Tenascin-C mRNA expression levels increased further following co-culture with mechanically stretched fibroblasts. These data suggested that the effects of TGF- $\beta 1$ and indirect co-culture with strained fibroblasts on tenascin- $\mathrm{C}$ expression in BMSCs were synergistic.

Induction of tenascin-C mRNA expression via TGF- $\beta 1$ and the MAPK signaling pathway. TGF- $\beta 1$ has been reported to be a potent growth factor with the capacity to induce tenascin-C expression in fibroblasts (33). If indirect co-culture with the stretched fibroblasts induces tenascin-C mRNA expression level in BMSCs via the paracrine release of TGF- $\beta 1$, the effects of mechanical stimulation and TGF- $\beta 1$ treatment may be mediated via an intracellular signaling pathway. To test this hypothesis, the effects of specific inhibitors on the increase of tenascin-C mRNA expression levels by TGF- $\beta 1$ and co-culture with mechanically stretched fibroblasts were investigated. Treatment with $5 \mathrm{ng} / \mathrm{ml}$ TGF- $\beta 1$ for 6 days led to a 2.4-fold increase in tenascin-C mRNA expression levels in BMSCs (Fig. 5). SB 431542 is a specific inhibitor of activin receptor-like kinase, the TGF- $\beta$ receptor type I. As presented in Fig. 5, $10 \mu \mathrm{M}$ SB 431542 inhibited the TGF- $\beta 1$-dependent increase of tenascin-C mRNA expression levels by $~ 59 \%$. A specific inhibitor of MEK-1, $10 \mu \mathrm{M}$ PD 98059 significantly decreased tenascin-C mRNA expression levels in BMSCs by $\sim 61 \%$. A similar inhibition was observed following treatment with $3 \mu \mathrm{M}$ SB 203580, a p38 MAPK inhibitor. These data indicate that activation of the MEK/p38 MAPK signaling pathway may contribute to the TGF- $\beta 1$-dependent increase in tenascin-C expression.

Mechanically stretched fibroblasts indirectly induce tenascin-C expression in BMSCs via the MEK/p38 MAPK signaling pathway. TGF- $\beta 1$-dependent induction of tenascin-C expression may require activation of $\mathrm{MEK} / \mathrm{p} 38 \mathrm{MAPK}$ in BMSCs. In addition, mechanical stretching significantly induced TGF- $\beta 1$ expression in stretched fibroblasts (Fig. 6A) and BMSCs co-cultured with stretched fibroblasts (Fig. 6B). Therefore, the involvement of the MEK/MAPK signaling pathway was investigated in the induction of tenascin- $\mathrm{C}$ expression in BMSCs by co-culture with mechanically 
A

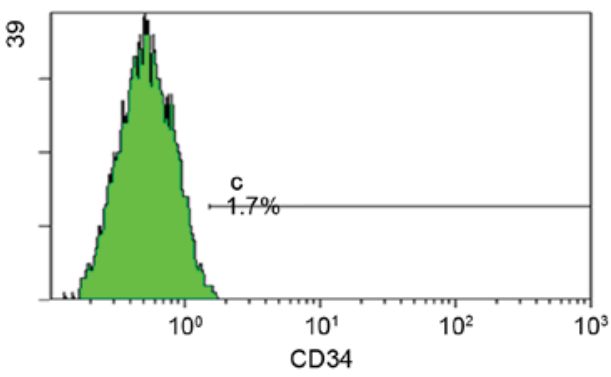

C

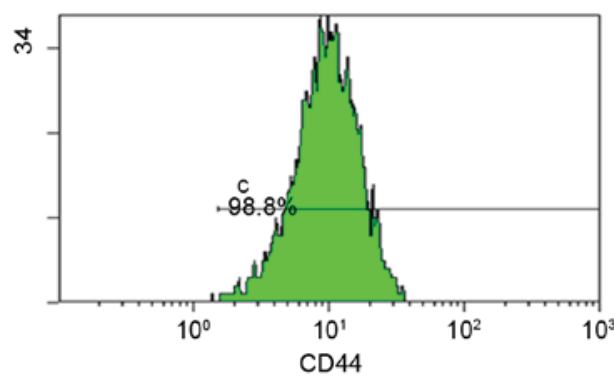

B

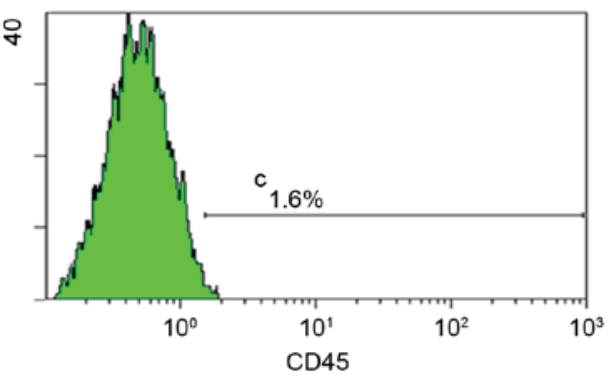

D

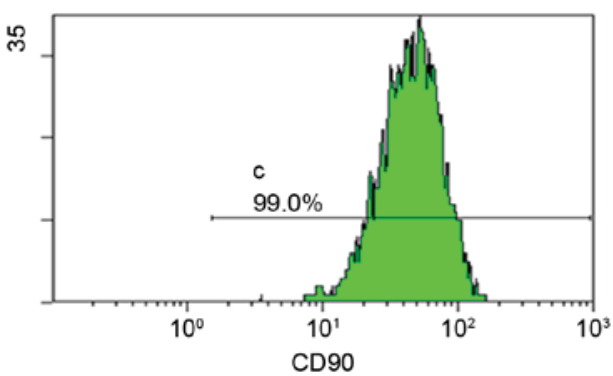

Figure 1. Expression profile of BMSCs. Representative flow cytometry histograms demonstrating the percentage of cells positive for the stem cell markers (A) CD34, (B) CD45, (C) CD44 and (D) CD90 in primary BMSCs at passage 4, isolated from 7-day-old Sprague Dawley rats. BMSCs, bone marrow mesenchymal stem cells; $\mathrm{CD}$, cluster of differentiation.

A

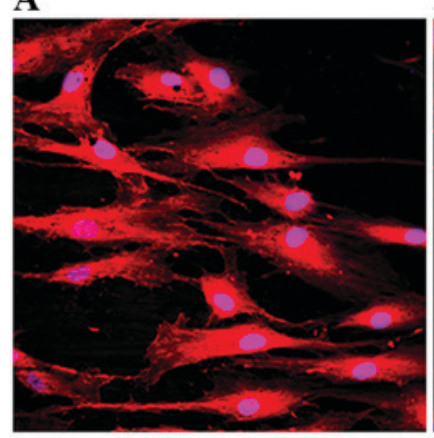

B

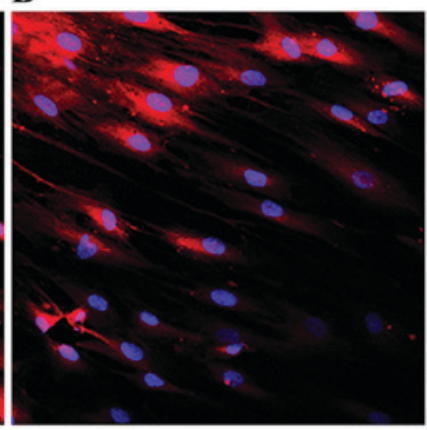

\section{C} $0 \mathrm{~h} \quad 3 \mathrm{~h} \quad 12 \mathrm{~h} \quad 24 \mathrm{~h}$

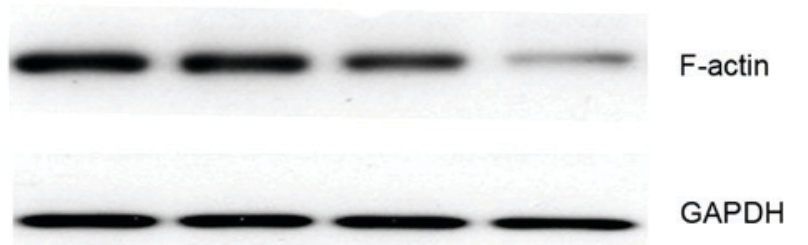

Figure 2. F-actin expression following mechanical stress. Laser confocal microscopy images of F-actin staining (red) following mechanical stress in rat ligament fibroblasts under $10 \%$ stretching at $1 \mathrm{~Hz}$ for (A) 0 and (B) $24 \mathrm{~h}$. Cells were counterstained with DAPI. (C) Protein expression levels of F-actin following cyclical stretching for $0,3,12$ or $24 \mathrm{~h}$, as assessed by western blotting. Representative images are presented. Magnification, $\mathrm{x} 400$.

stretched fibroblasts. As presented in Fig. 6C, the increase in tenascin-C mRNA expression levels induced by indirect co-culture with mechanically stretched fibroblasts was significantly inhibited by neutralizing anti-TGF- $\beta 1$ antibodies and SB 431542. Similarly, PD 98059 and SB 203580 significantly

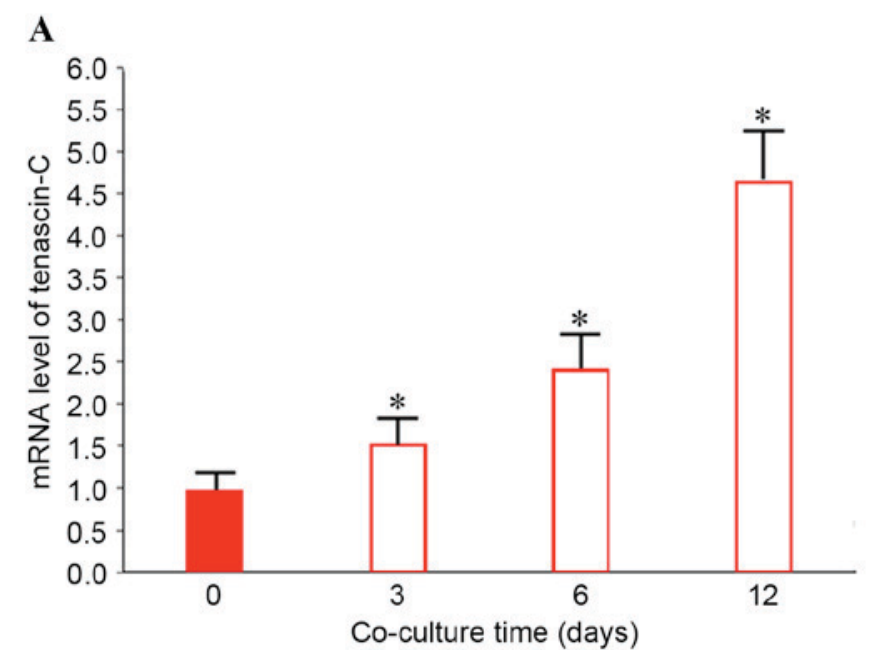

B

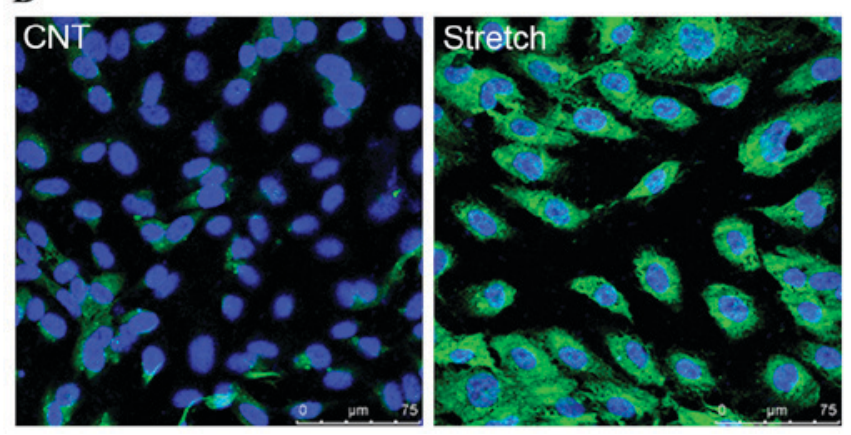

Figure 3. Fibroblasts were subjected to mechanical stretch for $12 \mathrm{~h}$ and BMSCs were co-cultured with fibroblasts for different time periods. (A) mRNA expression levels of tenascin-C in BMSCs, as determined by reverse transcription-quantitative polymerase chain reaction. Data are expressed as the mean \pm standard deviation, ${ }^{\prime} \mathrm{P}<0.05$ vs. day 0 . (B) Immunofluorescence staining of tenascin-C (green) in BMSCs following co-culture for 12 days with ligament fibroblasts without mechanical stretch (control, bottom left panel), or with mechanical stretch (bottom right panel). BMSCs, bone marrow mesenchymal stem cells; CNT, control. 


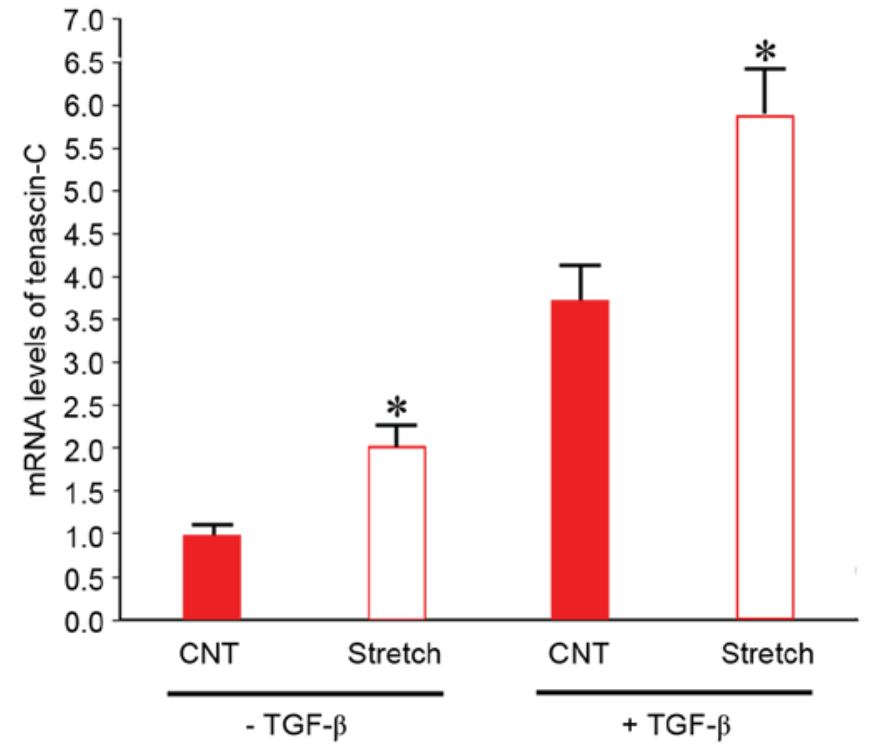

Figure 4. Synergistic effects of TGF- $\beta$ and indirect co-culture with mechanically stretched fibroblasts on tenascin-C expression. BMSCs were cultured in the absence or presence of $5 \mathrm{ng} / \mathrm{ml}$ TGF- $\beta 1$ for 6 days. These cells were co-cultured with ligament fibroblasts subject to stretching or control unmanipulated fibroblasts. Total RNA was extracted and subjected to reverse transcription-quantitative polymerase chain reaction to analyze the mRNA expression levels of tenascin-C. Data are expressed as the mean \pm standard deviation ( $n=3)$. $\mathrm{P}<0.05$ vs. BMSCs in respective CNT groups. TGF, transforming growth factor; BMSCs, bone marrow mesenchymal stem cells; CNT, control.

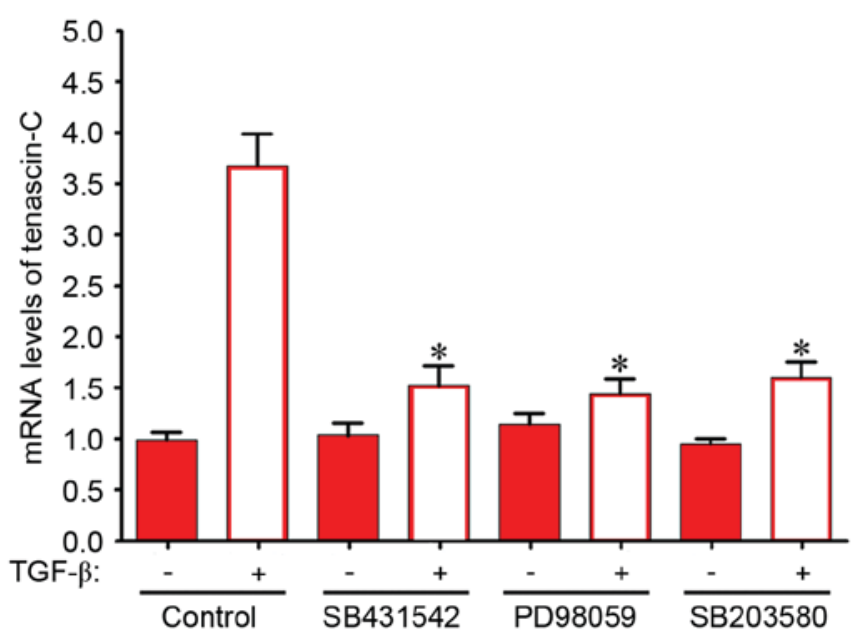

Figure 5. Increase in tenascin-C mRNA expression levels in BMSCs induced by TGF- $\beta$ is attenuated by inhibitors of TGF- $\beta$ receptor type I, MEK- 1 and p38 MAPK. BMSCs co-cultured with ligament fibroblasts were incubated in medium containing dimethyl sulfoxide (control), $10 \mu \mathrm{M} \mathrm{SB} 431542$ (a TGF- $\beta$ receptor type I inhibitor), $10 \mu \mathrm{M}$ PD 98059 (a MEK-1 inhibitor) or $3 \mu \mathrm{M}$ SB 203580 (a p38 MAPK inhibitor). Subsequently, TGF- $\beta 1$ was added and BMSCs were cultured for 6 days. Tenascin-C mRNA expression levels were analyzed using reverse transcription-quantitative polymerase chain reaction. Data are expressed as the mean \pm standard deviation $(n=5)$. ${ }^{*} \mathrm{P}<0.05$ vs. TGF- $\beta 1$ treatment in the absence of inhibitors. BMSCs, bone marrow mesenchymal stem cells; TGF, transforming growth factor; MEK, mitogen-activated protein kinase kinase; MAPK, mitogen-activated protein kinase.

attenuated the increase in tenascin- $\mathrm{C}$ expression levels in BMSCs. These results suggested that in the indirect co-culture
A

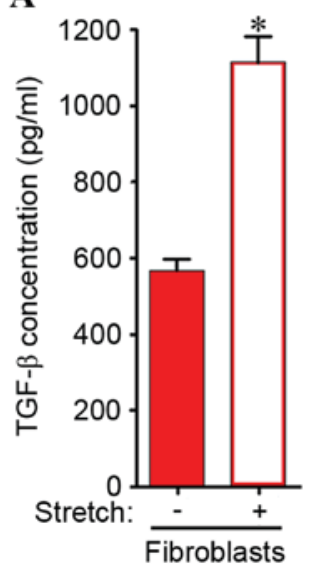

C

B

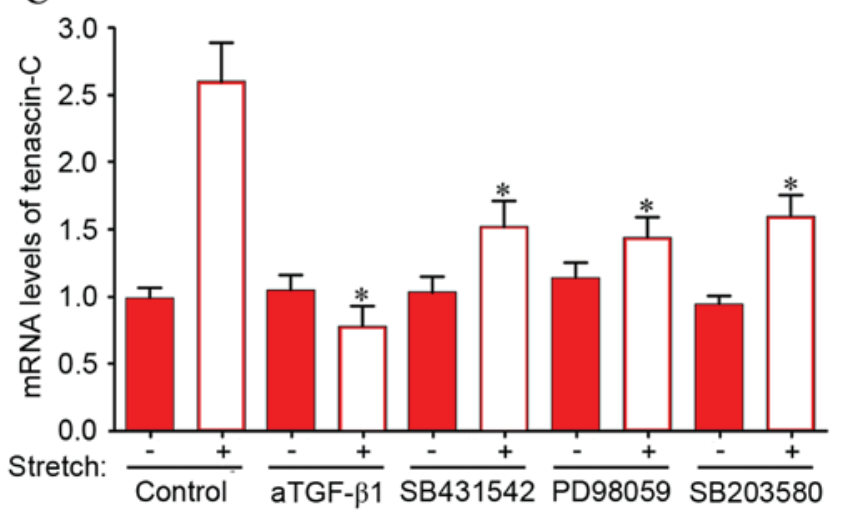

Figure 6. (A) Fibroblasts only were subjected to direct mechanical stretch with $10 \%$ load and $1 \mathrm{~Hz}$. (B) BMSCs were co-cultured with stretched ligament fibroblasts. An ELISA was used to examine the TGF- $\beta$ concentrations of stretched ligament fibroblasts, and BMSCs co-cultured with stretched ligament fibroblasts. Data are expressed as the mean \pm standard deviation. ${ }^{*} \mathrm{P}<0.05$ vs. unstretched control fibroblasts or BMSCs cultured with unstretched fibroblasts, respectively. (C) Increase in tenascin-C mRNA expression levels in BMSCs co-cultured with ligament fibroblasts was inhibited by neutralizing anti-TGF- $\beta 1$ antibodies and inhibitors of TGF- $\beta$ receptor type I, MEK-1 and p38 MAPK. BMSCs co-cultured with ligament fibroblasts were incubated in medium containing DMSO (control), $10 \mu \mathrm{M}$ SB 431542 (a TGF- $\beta$ receptor type I inhibitor), $10 \mu \mathrm{M}$ PD 98059 (a MEK-1 inhibitor) or $3 \mu \mathrm{M}$ SB 203580 (a p38 MAPK inhibitor). Fibroblasts were subjected to mechanical stretching with $10 \%$ load and $1 \mathrm{~Hz}$. Following 6 days of co-culture, BMSCs were collected for reverse transcription-quantitative polymerase chain reaction. Data are expressed as the mean \pm standard deviation $(n=5)$. $\mathrm{P}<0.05$ vs. stretched control in the absence of inhibitors. BMSCs, bone marrow mesenchymal stem cells; DMSO, dimethyl sulfoxide; TGF, transforming growth factor; MEK, mitogen-activated protein kinase kinase; MAPK, mitogen-activated protein kinase.

system, the intracellular MEK/p38 MAPK signaling pathway is responsible for the upregulation of tenascin- $\mathrm{C}$ expression levels induced by mechanical stretch stimulation.

\section{Discussion}

POP is a distressing morbidity that affects the quality of life of patients in developed and developing countries (34). Pelvic organ support is maintained by complex interactions between levator ani muscles and connective tissues of the urethra, vaginal wall and rectum. Abnormalities of normal levator ani function are a key feature of POP (35). Stem cells have the potential to develop into numerous different specialized 
cells in the body. There is increasing evidence to suggest that BMSCs may be used as a novel cell-based therapy to repair ligament, tendon and cartilage damage (36).

The present study demonstrated that $>95 \%$ of BMSCs expressed CD90 and CD44, and these BMSCs may differentiate into osteogenic and adipogenic cells. Therefore, the isolated primary BMSCs in the present study possessed the characteristics of stem cells. Our previous study revealed that indirect mechanical stretching alters cell morphology and arrangement, and stimulates the expression and secretion of ECM components including type I and III collagen, elastin, lysyl oxidase and fibulin-5 in co-cultured BMSCs (26). This indicated that co-culture with mechanically stretched ligament fibroblasts promotes BMSC differentiation into fibroblasts, consistent with a separate report $(9,37)$. Previous studies have reported that tenascin- $\mathrm{C}$ expression is significantly increased in prolapsed ligaments $(34,38)$. As an ECM protein, tenascin-C is transiently present in the ECM and is markedly upregulated in tissue repair. Regulation of tenascin- $\mathrm{C}$ gene expression is complex and may be involved in the process of BMSC differentiation. However, the molecular mechanisms underlying the regulation of tenascin- $\mathrm{C}$ expression in various conditions remain to be fully elucidated. Therefore, the present study investigated the mechanisms via which indirect mechanical stretching regulates tenascin-C expression in BMSCs.

Mechanical stretching has been reported to indirectly induce expression of ECM components (37). A mechanical signal has been demonstrated to trigger the release of growth factors in an auto- or paracrine manner in numerous cell types (39). These factors modify the transcription rate of specific downstream genes including tenascin- $\mathrm{C}$ and other matrix ingredients. A previous study has revealed that tenascin- $\mathrm{C}$ expression levels are affected by various growth factors including TGF- $\beta$, platelet-derived growth factor and certain cytokines (40). TGF- $\beta$ may stimulate the production of ECM proteins by fibroblasts. Although mechanical stretching is an important regulator of tenascin- $\mathrm{C}$ expression, it may act indirectly to induce tenascin- $\mathrm{C}$ expression via auto- or paracrine release of growth factors. For example, release of TGF- $\beta$ was induced by cyclic stretching in cardiac fibroblasts, and was required to promote pro-collagen $\alpha 1$ (41). Therefore, TGF- $\beta$ may indirectly mediate the response to mechanical stimulation.

The results of the present study indicated that mechanical stretching indirectly increased tenascin- $\mathrm{C}$ mRNA expression levels in BMSCs, potentially via TGF- $\beta$, the production of which was induced by stretching. The increase in tenascin- $\mathrm{C}$ expression levels induced by mechanical stress was synergistic with that induced by TGF- $\beta$ treatment. Investigation of gene induction by mechanical stretching has primarily been performed using serum free medium (26). The present study revealed that mechanical stretching-induced tenascin- $\mathrm{C}$ mRNA expression may be further increased by TGF- $\beta$ treatment. TGF- $\beta$ signaling is primarily mediated by the mothers against decapentaplegic (SMAD)-dependent pathway. However, TGF- $\beta$ has been reported to additionally activate the MAPK signaling pathway, which is SMAD-independent, and has been demonstrated to induce the expression of ECM components with the involvement of the TGF- $\beta$ /MAPK pathway (42). Thus, TGF- $\beta 1$ and mechanical stretching-induced TGF- $\beta 1$ may be inhibited by SB 431542 for the direct and indirect induction of tenascin-C expression. In addition, specific inhibitors of MEK and MAPK significantly inhibited the increase in tenascin- $C$ mRNA expression levels induced by TGF- $\beta 1$ and indirect mechanical stretching. These results suggested that regulation of tenascin-C expression levels in BMSCs co-cultured with mechanically stretched pelvic ligament fibroblasts is mediated via TGF- $\beta 1$ and MEK/MAPK pathways.

The present study demonstrated that indirect co-culture with mechanically stretched fibroblasts increased tenascin- $\mathrm{C}$ expression levels in BMSCs. Consistent with this, it has been reported that following co-culture with ligament fibroblasts for 5 days, BMSCs may differentiate into fibroblasts (37). Conversely, a previous study has demonstrated that indirect co-culture has no effect on the differentiation of BMSCs (43). The results of the present study suggested that indirect co-culture is important for the differentiation of BMSCs as mechanically stretched fibroblasts may produce soluble factors that activate and regulate intracellular signaling pathways in the indirect co-cultured BMSCs. The present study suggested that BMSCs may be used as a potential novel cell-based therapy for the treatment of POP and other diseases. Future studies aim to investigate the detailed underlying mechanisms of intracellular pathways in the differentiation of BMSCs co-cultured with injured ligament fibroblasts.

\section{Acknowledgements}

The present study was supported by the National Natural Science Foundation of China (grant no. 81300469) and the Key Technology Research and Development Program Foundation of Henan Provincial Health Bureau (grant no. 201303093).

\section{References}

1. Choi KH and Hong JY: Management of pelvic organ prolapse. Korean J Urol 55: 693-702, 2014.

2. Giarenis I and Robinson D: Prevention and management of pelvic organ prolapse. F1000Prime Rep 6: 77, 2014.

3. Wiegersma M, Panman CM, Kollen BJ, Berger MY, Lisman-Van Leeuwen Y and Dekker JH: Effect of pelvic floor muscle training compared with watchful waiting in older women with symptomatic mild pelvic organ prolapse: Randomised controlled trial in primary care. BMJ 349: g7378, 2014.

4. Zhou Y, Ling O and Bo L: Expression and significance of lysyl oxidase-like 1 and fibulin-5 in the cardinal ligament tissue of patients with pelvic floor dysfunction. J Biomed Res 27: 23-28, 2013.

5. Aznal SS, Meng FG, Nalliah S, Tay A, Chinniah K and Jamli MF: Biochemical evaluation of the supporting structure of pelvic organs in selected numbers of premenopausal and postmenopausal Malaysian women. Indian J Pathol Microbiol 55: 450-455, 2012.

6. Wynn RF, Hart CA, Corradi-Perini C, O'Neill L, Evans CA, Wraith JE, Fairbairn LJ and Bellantuono I: A small proportion of mesenchymal stem cells strongly expresses functionally active CXCR4 receptor capable of promoting migration to bone marrow. Blood 104: 2643-2645, 2004.

7. Xu YX, Chen L, Hou WK, Lin P, Sun L, Sun Y, Dong QY, Liu JB and Fu YL: Mesenchymal stem cells treated with rat pancreatic extract secrete cytokines that improve the glycometabolism of diabetic rats. Transplant Proc 41: 1878-1884, 2009.

8. Miller MD, Nichols T and Butler CA: Patella fracture and proximal patellar tendon rupture following arthroscopic anterior cruciate ligament reconstruction. Arthroscopy 15: 640-643, 1999. 
9. Omoto M, Miyashita H, Shimmura S, Higa K, Kawakita T, Yoshida S, McGrogan M, Shimazaki J and Tsubota K: The use of human mesenchymal stem cell-derived feeder cells for the cultivation of transplantable epithelial sheets. Invest Ophthalmol Vis Sci 50: 2109-2115, 2009.

10. Young RG, Butler DL, Weber W, Caplan AI, Gordon SL and Fink DJ: Use of mesenchymal stem cells in a collagen matrix for Achilles tendon repair. J Orthop Res 16: 406-413, 1998.

11. Hankemeier S, Hurschler C, Zeichen J, van Griensven M, Miller B, Meller R, Ezechieli M, Krettek C and Jagodzinski M: Bone marrow stromal cells in a liquid fibrin matrix improve the healing process of patellar tendon window defects. Tissue Eng Part A 15: 1019-1030, 2009.

12. Kushida $\mathrm{T}$ and Iida $\mathrm{H}$ : Bone marrow cell transplantation efficiently repairs tendon and ligament injuries. Front Cell Dev Biol 2: 27, 2014.

13. Humphrey JD, Dufresne ER and Schwartz MA: Mechanotransduction and extracellular matrix homeostasis. Nat Rev Mol Cell Biol 15: 802-812, 2014.

14. Provenzano PP and Keely PJ: Mechanical signaling through the cytoskeleton regulates cell proliferation by coordinated focal adhesion and Rho GTPase signaling. J Cell Sci 124: 1195-1205, 2011.

15. Hwang Y and Barakat AI: Dynamics of mechanical signal transmission through prestressed stress fibers. PLoS One 7: e35343, 2012.

16. Horiguchi M, Ota M and Rifkin DB: Matrix control of transforming growth factor- $\beta$ function. J Biochem 152: 321-329, 2012.

17. Midwood KS and Orend G: The role of tenascin- $\mathrm{C}$ in tissue injury and tumorigenesis. J Cell Commun Signal 3: 287-310, 2009.

18. Mackie EJ, Halfter W and Liverani D: Induction of tenascin in healing wounds. J Cell Biol 107: 2757-2767, 1988.

19. Imanaka-Yoshida $\mathrm{K}$ and Aoki $\mathrm{H}$ : Tenascin- $\mathrm{C}$ and mechanotransduction in the development and diseases of cardiovascular system. Front Physiol 5: 283, 2014

20. Wang SJ, Zhu B, Ren XX, Ben H, Li YQ and Li YH: Effect of acupuncture of different acupoints on electrical activities of hypothalamic sexual arousal stimulation-related neurons at different stages of oestrous cycle in rats. Zhen Ci Yan Jiu 32: 313-318, 2007 (In Chinese)

21. Bing Z, Linlin L, Jianguo Y, Shenshen R, Ruifang R and Xi Z: Effect of mechanical stretch on the expressions of elastin, LOX and Fibulin-5 in rat BMSCs with ligament fibroblasts co-culture. Mol Biol Rep 39: 6077-6085, 2012.

22. Zaminy A, Ragerdi Kashani I, Barbarestani M, Hedayatpour A, Mahmoudi R and Farzaneh Nejad A: Osteogenic differentiation of rat mesenchymal stem cells from adipose tissue in comparison with bone marrow mesenchymal stem cells: Melatonin as a differentiation factor. Iran Biomed J 12: 133-141, 2008.

23. Jeong JY, Suresh S, Park MN, Jang M, Park S, Gobianand K, You S, Yeon SH and Lee HJ: Effects of capsaicin on adipogenic differentiation in bovine bone marrow mesenchymal stem cell. Asian-Australas J Anim Sci 27: 1783-1793, 2014

24. Wilson E, Mai Q, Sudhir K, Weiss RH and Ives HE: Mechanical strain induces growth of vascular smooth muscle cells via autocrine action of PDGF. J Cell Biol 123: 741-747, 1993.

25. Millward-Sadler SJ, Wright MO, Lee H, Nishida K, Caldwell H, Nuki G and Salter DM: Integrin-regulated secretion of interleukin 4: A novel pathway of mechanotransduction in human articular chondrocytes. J Cell Biol 145: 183-189, 1999.
26. Yamamoto K, Dang QN, Kennedy SP, Osathanondh R, Kelly RA and Lee RT: Induction of tenascin-C in cardiac myocytes by mechanical deformation. Role of reactive oxygen species. J Biol Chem 274: 21840-21846, 1999.

27. Heid CA, Stevens J, Livak KJ and Williams PM: Real time quantitative PCR. Genome Res 6: 986-994, 1996.

28. Gibson UE, Heid CA and Williams PM: A novel method for real time quantitative RT-PCR. Genome Res 6: 995-1001, 1996.

29. Livak KJ and Schmittgen TD: Analysis of relative gene expression data using real-time quantitative PCR and the 2(-Delta Delta C(T)) Method. Methods 25: 402-408, 2001.

30. Ren CC, Ren RF, Zhao B, Zhang X and Jiang YJ: Study on oriented differentiation of bone marrow mesenchymal stem cells by fibroblast in rat uterine ligament with mechanical stretch. Zhonghua Fu Chan Ke Za Zhi 46: 527-532, 2011 (In Chinese).

31. Wang JH, Thampatty BP, Lin JS and Im HJ: Mechanoregulation of gene expression in fibroblasts. Gene 391: 1-15, 2007.

32. Chiquet M, Gelman L, Lutz R and Maier S: From mechanotransduction to extracellular matrix gene expression in fibroblasts. Biochim Biophys Acta 1793: 911-920, 2009.

33. Jinnin M, Ihn H, Asano Y, Yamane K, Trojanowska M and Tamaki K: Tenascin-C upregulation by transforming growth factor-beta in human dermal fibroblasts involves Smad3, Sp1, and Ets1. Oncogene 23: 1656-1667, 2004

34. Ewies AA, Al-Azzawi F and Thompson J: Changes in extracellular matrix proteins in the cardinal ligaments of post-menopausal women with or without prolapse: A computerized immunohistomorphometric analysis. Hum Reprod 18: 2189-2195, 2003.

35. Boreham MK, Wai CY, Miller RT, Schaffer JI and Word RA Morphometric properties of the posterior vaginal wall in women with pelvic organ prolapse. Am J Obstet Gynecol 187: 1501-1509, 2002.

36. Kon E, Filardo G, Roffi A, Andriolo L and Marcacci M: New trends for knee cartilage regeneration: From cell-free scaffolds to mesenchymal stem cells. Curr Rev Musculoskelet Med 5: 236-243, 2012

37. Lee IC, Wang JH, Lee YT and Young TH: The differentiation of mesenchymal stem cells by mechanical stress or/and co-culture system. Biochem Biophys Res Commun 352: 147-152, 2007.

38. Goepel C: Differential elastin and tenascin immunolabeling in the uterosacral ligaments in postmenopausal women with and without pelvic organ prolapse. Acta Histochem 110: 204-209, 2008.

39. Chiquet M, Sarasa-Renedo A and Tunc-Civelek V: Induction of tenascin-C by cyclic tensile strain versus growth factors: Distinct contributions by Rho/ROCK and MAPK signaling pathways. Biochim Biophys Acta 1693: 193-204, 2004.

40. Chiquet-Ehrismann R and Chiquet M: Tenascins: Regulation and putative functions during pathological stress. J Pathol 200: 488-499, 2003.

41. Lindahl GE, Chambers RC, Papakrivopoulou J, Dawson SJ, Jacobsen MC, Bishop JE and Laurent GJ: Activation of fibroblast procollagen alpha 1(I) transcription by mechanical strain is transforming growth factor-beta-dependent and involves increased binding of CCAAT-binding factor $(\mathrm{CBF} / \mathrm{NF}-\mathrm{Y})$ at the proximal promoter. J Biol Chem 277: 6153-6161, 2002.

42. Zhang YE: Non-Smad pathways in TGF-beta signaling. Cell Res 19: 128-139, 2009.

43. Li H, Yu B, Zhang Y, Pan Z, Xu W and Li H: Jagged1 protein enhances the differentiation of mesenchymal stem cells into cardiomyocytes. Biochem Biophys Res Commun 341: 320-325, 2006. 